\title{
Treatment patterns, healthcare resource utilization, and costs following first-line antidepressant treatment in major depressive disorder: a retrospective US claims database analysis
}

Geneviève Gauthier ${ }^{1}$, Annie Guérin ${ }^{1}$, Maryia Zhdanava ${ }^{1}$, William Jacobson², George Nomikos ${ }^{3}$, Elizabeth Merikle ${ }^{4}$, Clément François ${ }^{5}$ and Vanessa Perez ${ }^{2^{*}}$ (D)

\begin{abstract}
Background: Although the symptoms of major depressive disorder (MDD) are often manageable with pharmacotherapy, response to first-line antidepressant treatment is often less than optimal. This study describes long-term treatment patterns in MDD patients in the United States and quantifies the economic burden associated with different treatment patterns following first-line antidepressant therapy.

Methods: MDD patients starting first-line antidepressant monotherapy and having continuous enrollment $\geq 12$ months before and $\geq 24$ months following the index date (i.e., the first documented prescription fill) were selected from the Truven Health Analytics MarketScan (2003-2014) database. Based on the type of first treatment change following initiation, six treatment cohorts were defined a priori ("persistence"; "discontinuation"; "switch"; "dose escalation"; "augmentation"; and "combination"). Treatment patterns through the fourth line of therapy within each cohort, healthcare resource utilization (HCRU), and cost analyses were restricted to patients with adequate treatment duration (defined as $\geq 42$ days) in each line (analysis sub-sample, $N=21,088$ ). HCRU and costs were described at the cohort and pattern levels. Treatment cohorts representing $<5 \%$ of the analysis sub-sample were decided a priori not to be analyzed due to limited sample size.
\end{abstract}

Results: 39,557 patients were included. Mean age was 42.1 years, $61.1 \%$ of patients were female, and mean follow-up was 4.1 years. Among the analysis sub-sample, the discontinuation (49.1\%), dose escalation (37.4\%), and switch (6.6\%) cohorts were the most common of all treatment cohorts. First-line antidepressant discontinuation without subsequent MDD pharmacotherapy (22.9\%) and cycling between discontinuation and resumption (11.2\%) were the two most common treatment patterns. Median time to discontinuation was 23 weeks. The switch cohort exhibited the highest HCRU (18.9 days with medical visits per-patient-per-year) and greatest healthcare costs $(\$ 11,107$ per-patient-per-year) following the index date. Treatment patterns representing a cycling on and off treatment in the switch cohort were associated with the greatest healthcare costs overall. (Continued on next page)

\footnotetext{
* Correspondence: Vanessa.Perez@takeda.com

${ }^{2}$ Takeda Development Center Americas, Inc., One Takeda Parkway, Deerfield,

IL 60015, USA

Full list of author information is available at the end of the article
} 
(Continued from previous page)

Conclusion: A high proportion of patients discontinue first-line antidepressant shortly after initiation. Patterns representing a cycling on and off treatment in the switch cohort were associated with the highest healthcare costs. These findings underscore challenges in effectively treating patients with MDD and a need for personalized patient management.

Keywords: Major depressive disorder, Antidepressants, Treatment patterns, Economic burden, Healthcare resource utilization, Healthcare costs

\section{Background}

Major depressive disorder (MDD) is a complex, multifaceted psychiatric condition characterized by a variety of symptoms, including a persistent state of sadness and hopelessness, anhedonia, sleep disturbance, indecision, reduced ability to concentrate, and recurrent suicidal ideation [1,2]. MDD is one of the most prevalent mental disorders in the United States (US) [3] and a leading cause of global disability [4]. According to the 2014 National Survey on Drug Use and Health, $6.6 \%$ of all adults, or 15.7 million people, in the US experienced at least one major depressive episode in the prior 12-month period [5]. The high prevalence and significant functional impact of MDD [6-9] result in considerable economic consequences at all levels of society. Recent evidence shows that the total economic burden of MDD in the US has increased notably in the last decade, up to $\$ 210$ billion dollars in 2010, with workplace and healthcare costs contributing almost equally ( $48 \%$ and $47 \%$, respectively) followed by suicide-related costs (5\%) [10].

Symptoms of MDD are often managed by appropriate psychological and/or pharmacological therapy and should be addressed throughout the depression life-course [11]. Practice guidelines recommend that treatments be individually tailored and based on disease severity and history, level of functioning, and comorbid psychiatric and physical conditions $[6,7,12,13]$. Antidepressant drugs constitute the standard of care for $\operatorname{MDD}[6,7,14]$, whereby most patients will receive a selective serotonin reuptake inhibitor (SSRI) as first-line pharmacotherapy [15]. However, response to first-line antidepressant treatment is often not optimal [16-19].

Management of MDD following non-optimal response to pharmacotherapy can be summarized by four key treatment steps as presented in current US guidelines: (1) dose optimization to maximize therapeutic benefit, (2) therapy switch (i.e., discontinuation of the current pharmacotherapy in favor of an alternative pharmacotherapy), (3) combination therapy (i.e., adding a second antidepressant drug to the initial one), or (4) augmentation therapy (i.e., augmenting the initial antidepressant drug with a nonantidepressant pharmacotherapy) $[11,14,18]$. Treatment regimens prescribed in the real-world setting do not always conform to practice guidelines or on-label use of treatment for MDD. Therapeutic decisions are influenced by a number of factors including safety and tolerability, adherence, and patient-physician dynamics [20]. Nonetheless, identification of the most suitable treatment for each patient as early as possible remains a key challenge and represents a barrier to the improvement of long-term treatment outcomes in MDD $[7,20]$.

An assessment of long-term real-world treatment patterns in MDD can provide empirical evidence on patient burden and illustrate the economic impact associated with different pharmacological approaches taken following initiation of first-line therapy. In this study, we describe long-term real-world treatment patterns among MDD patients in the US and quantify healthcare resource utilization (HCRU) and costs associated with different treatment strategies following first-line antidepressant therapy.

\section{Methods}

\section{Data source}

This study used data from the Truven Health Analytics MarketScan ${ }^{\circ}$ Database (MarketScan) [21] between January 2003 through March 2014. The MarketScan database is a large US private sector health claims database containing enrollment history, pharmacy and medical (professional and institutional) claims of employees and their dependents, as well as Medicare-eligible retirees with employer-provided Medicare supplemental plans. The database covers all census regions of the United States. MarketScan data are de-identified and comply with the Health Insurance Portability and Accountability Act [22]. No institutional review board approval was necessary for the current study.

\section{Study population and design}

Patients were included if they had at least two claims, within a 6-month period, containing a diagnosis for MDD (ICD-9 codes: $296.2 \times, 296.3 \times$ ) and initiated a firstline antidepressant as monotherapy on the same day or within 30 days of a claim containing a diagnosis for MDD. The index date was defined as the first documented prescription fill date for an antidepressant over the period covered by the database and was considered a proxy for first-line antidepressant initiation. Patients were 
required to be $\geq 18$ years of age as of the index date and continuously enrolled in a healthcare plan for $\geq 12$ months before and $\geq 24$ months after the index date. Patients were excluded if claims for a second antidepressant or an atypical antipsychotic were present within 30 days of the index date. In addition, patients with claims containing a diagnosis for any type of schizophrenic disorder (ICD-9 codes 295.xx) before or after the index date were excluded.

This study was retrospective in design (Fig. 1). The baseline period was defined by the 12-month period before the index date. The study period was defined by the period of continuous healthcare plan enrollment following the index date. A minimum study period of 24 months was required a priori for each patient in order to assess long-term treatment patterns.

\section{Study measures and treatment outcomes Baseline study characteristics}

Sociodemographics, healthcare plan information, the presence of physical and mental comorbidities, all-cause HCRU, number of visits to a mental health professional, and total healthcare costs during the baseline period were summarized among all patients meeting inclusion criteria.

\section{Treatment cohorts and patterns}

Treatment cohorts were defined based on the type of first treatment change following first-line antidepressant initiation, which included the four key treatment steps for patients with non-optimal response to antidepressant treatment as presented in current US guidelines: dose optimization, switch, combination treatment, and augmentation. For completeness, the present study also examined treatment discontinuation and persistence of initial antidepressant therapy. More specifically, six mutually exclusive cohorts were examined in the present study: (1) discontinuation cohort (i.e., patients with an interruption of $\geq 42$ consecutive days of the pharmacotherapy initiated at the beginning of the line of therapy),
(2) dose escalation cohort (i.e., patients with an increase in daily dose $\geq 50 \%$ compared with the prior dose), (3) switch cohort (i.e., patients who initiated a new drug regimen within 42 days of the discontinuation of the prior pharmacotherapy), (4) persistence cohort (i.e., patients who continued the first-line antidepressant therapy until the end of the study period), (5) combination cohort (i.e., patients who added an antidepressant to the initial antidepressant), and (6) augmentation cohort (i.e., patients who added an atypical antipsychotic to initial antidepressant). Definitions for each treatment cohort are presented in Table 1.

Each treatment cohort with the exception of the persistence cohort was further evaluated based on observed treatment patterns through the fourth line of therapy. Subsequent treatment changes from second through fourth line of therapy were identified in a similar manner, and, also included 'switch/drop' (note, this represented a treatment change in the overall drug regimen in the combination and augmentation cohorts only) and 'resumption' of pharmacotherapy (i.e., initiation of a new drug regimen or re-initiation of the same drug regimen following discontinuation). Each line of therapy was evaluated in terms of adequate treatment duration [11]. In this study, adequate treatment duration was defined as a minimum of 42 days before the occurrence of a treatment change.

The patient sample for analysis was restricted a priori to patients with adequate treatment duration in each line of therapy (analysis sub-sample).

\section{Analysis of healthcare resource utilization and costs}

Descriptive information on HCRU and costs was reported at the cohort level. Treatment cohorts representing $<5 \%$ of the analysis sub-sample were not analyzed due to limited sample size, a decision that was made a priori. Within each cohort included in the analysis, HCRU and costs were also examined at the treatment pattern level for the three most common patterns throughout the study period.

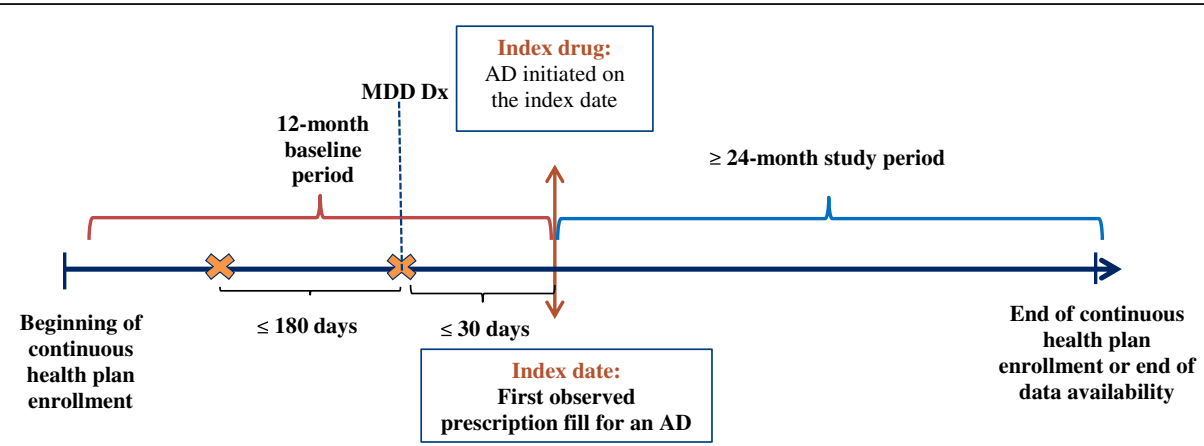

Fig. 1 Study design. ${ }^{*} M D D=$ major depressive disorder; $D x=$ diagnosis; $A D=$ antidepressant 
Table 1 First pharmacotherapy change definition

\begin{tabular}{|c|c|}
\hline 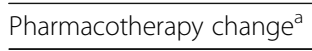 & Definition \\
\hline Discontinuation & $\begin{array}{l}\text { Interruption of } \geq 42 \text { consecutive days of the drug regimen initiated at the beginning of the line of } \\
\text { therapy }\end{array}$ \\
\hline Dose escalation & $\begin{array}{l}\text { Increase in daily dose of } \geq 50 \% \text { compared to prior dose - } \\
\text { A dose escalation occurring during the first } 21 \text { days was not considered in analyses as this was likely } \\
\text { due to titration for tolerability reasons. }\end{array}$ \\
\hline Switch $^{\text {b }}$ & $\begin{array}{l}\text { Initiation of a new drug regimen (antidepressant and/or atypical antipsychotic) within } 42 \text { days of the } \\
\text { discontinuation of the drug regimen initiated at the beginning of the line of therapy - Patients on } \\
\text { combination therapy at the beginning of a line of therapy who discontinued one treatment but remained } \\
\text { on the other treatment were deemed to have switched to a new drug regimen. }\end{array}$ \\
\hline Persistence & Absence of any of the treatment changes until the end of the study period \\
\hline Combination & Treatment add-on resulting in the use of $\geq 2$ antidepressants simultaneously. \\
\hline Augmentation & $\begin{array}{l}\text { Treatment add-on resulting in the use of one antidepressant and an atypical antipsychotic } \\
\text { simultaneously. }\end{array}$ \\
\hline
\end{tabular}

The mean and median total number of days with medical visits was estimated during the study period and reported per-patient-per-year (PPPY). The total number of days with medical visits was defined as the sum of inpatient days and days with emergency room visits, outpatient visits, and other medical services (i.e., laboratory, radiology, or other ancillary services). The proportion of the total number of days with mental health-related medical services (i.e., services associated with a claim containing an ICD-9 diagnosis code between 290.xx and 319.xx) was also reported.

The mean and median total healthcare costs incurred during the study period were reported PPPY. The total healthcare cost was defined as the sum of medical costs (combined inpatient, emergency room, outpatient, and other medical service costs) and pharmacy costs (MDDrelated pharmacy costs plus other pharmacy costs). The proportion of the total healthcare cost attributable to mental health-related medical services (i.e., medical costs associated with a claim containing an ICD-9 diagnosis code between 290.xx and 319.xx) and MDD-related pharmacy costs (i.e., total pharmacy costs for antidepressants and atypical antipsychotics) were also reported. Healthcare costs were reported in 2014 USD (adjusted for inflation using the Consumer Price Index for medical components) and reflect the total amount reimbursed by insurers and the out-of-pocket costs incurred by patients.

\section{Results}

\section{Baseline demographic and patient characteristics}

Inclusion criteria and the number of patients meeting each criterion are presented in Fig. 2. A total of 39,557 MDD patients met all the inclusion criteria and constituted the overall study sample (Fig. 2). The average age was 42.1 years $(\mathrm{SD}=15.1)$ and $61.1 \%$ of the overall study sample was female. Most patients were from the western (30.4\%) and southern (29.7\%) regions of the US and were insured by a preferred provider organization (PPO; 52.4\%) (Table 2). Only 2812 patients (7.1\%) were covered by a Medicare Supplemental plan at the index date. The prevalence of comorbid conditions was low (average Charlson-Quan Comorbidity Index score $=0.4 \pm 1.0$ ). Patients had, on average, a total of two visits to a mental health professional, one outpatient visit per month, and incurred an average annual total healthcare cost of $\$ 9941$ USD during the baseline period. SSRIs were the most common class of antidepressant drugs initiated on the index date $(69.5 \%$ of patients). Mean follow-up duration after the index date was 4.1 years $(\mathrm{SD}=1.9)$.

\section{Treatment cohorts and patterns}

A total of 21,088 patients had at least 42 days of treatment duration for each line of therapy evaluated and constituted the analysis sub-sample (Table 3) treatment duration of at least 42 days was considered a proxy for adequate treatment duration in this study [11]. The discontinuation, dose escalation, and switch cohorts were the most common treatment cohorts (discontinuation, 49.1\%; dose escalation, 37.4\%; and switch, 6.6\%, respectively). The two most common treatment patterns within the discontinuation cohort were discontinuation with no subsequent MDD-related pharmacotherapy (22.9\%) and cycling between therapy discontinuation and resumption (11.2\%; Table 3) - overall, after discontinuing the initial antidepressant, $16.7 \%$ of the patients later resumed one of the studied treatments (Table 3, pattern $1 \mathrm{~b}$ and 1c). Among patients who discontinued, median time to discontinuation of first-line therapy was 23 weeks. Cycling between therapy discontinuation and 


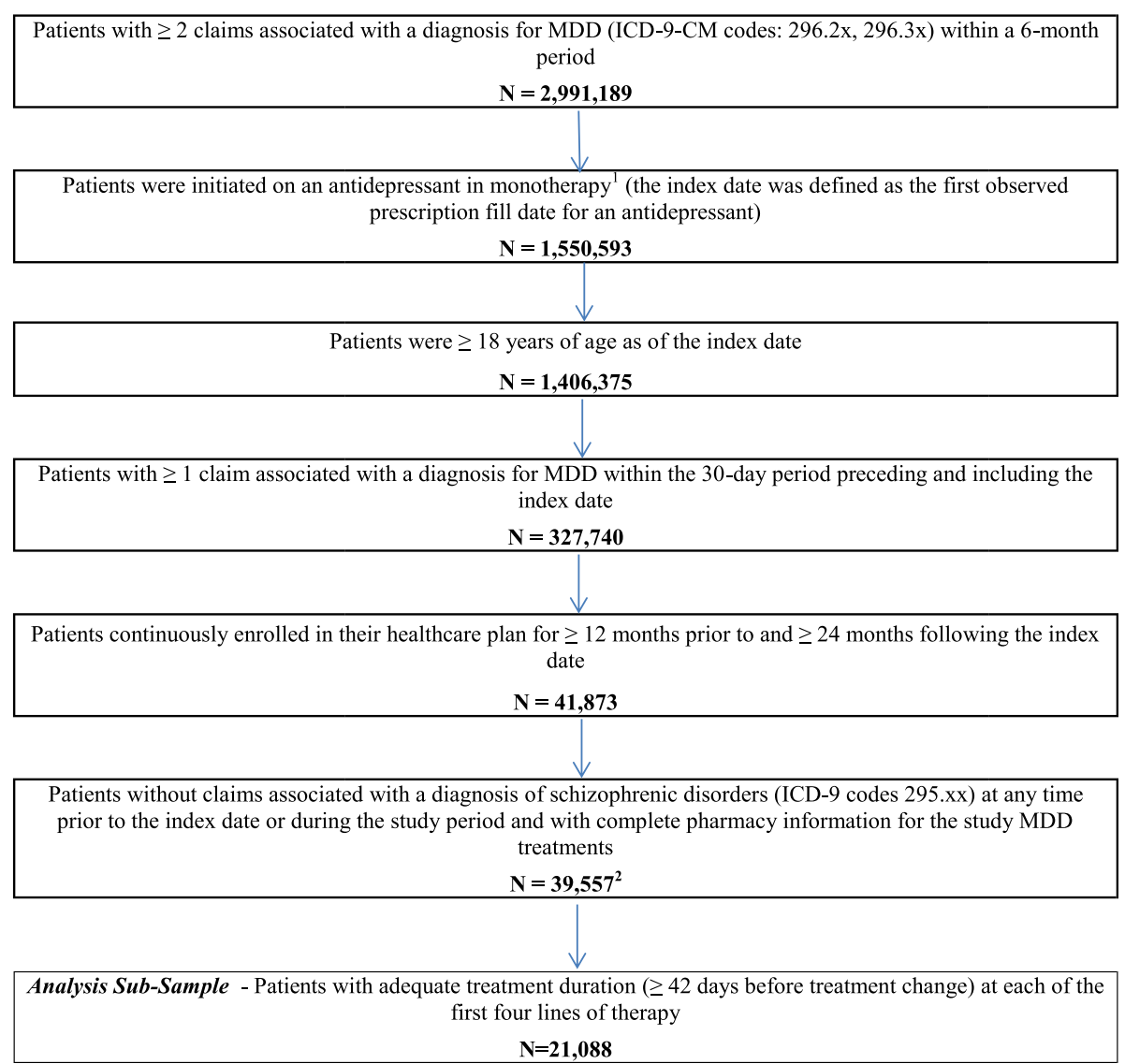

Fig. 2 Sample selection flowchart. ${ }^{*} M D D=$ major depressive disorder. [1] Patients were considered to be on monotherapy if they did not have any claims for another antidepressant or an atypical antipsychotic during the first 30 days following the index date. [2] Overall study sample without restriction in terms of adequate treatment duration

resumption following initial dose escalation was also one of the most common treatment patterns in the dose escalation cohort. Within the switch cohort, the most common treatment pattern was a switch from initial therapy followed by therapy discontinuation without further MDD-related pharmacotherapy (Table 3).

\section{Healthcare resource utilization and costs Treatment cohort-level}

HCRU PPPY and costs PPPY for the discontinuation, dose escalation, and switch cohorts, which each comprised more than $5 \%$ of the analysis sub-sample, are presented in Fig. 3a and b, respectively. During the study period, the switch cohort exhibited the highest level of HCRU on average (18.9 days with medical visits PPPY; Fig. 3a). Less than half (38.1\%) of these visits were mental health-related. Similarly, the switch cohort incurred the greatest amount of total healthcare costs, on average, during the study period (\$11,107 PPPY; Fig. 3b), and less than a quarter $(16.5 \%)$ of the total healthcare costs were mental health-related.

\section{Treatment pattern-level}

On average, HCRU was the highest among patients who escalated the dose of their first-line therapy and remained on the antidepressant until the end of the study period (pattern 2c in the dose escalation cohort: 19.2 days with medical visits, on average, PPPY; Fig. 4a). This estimate was similar to the average number of days with medical visits among patients who switched from their index antidepressant and then cycled on and off treatment (pattern 3b in the switch cohort: 19.1 days with medical visits, on average, PPPY; Fig. 4a). Mental health-related medical services accounted for less than half of the overall HCRU in these two treatment patterns (Fig. 4a).

Among the discontinuation, dose escalation, and switch cohorts, average total healthcare costs were highest for patients in the switch cohort who switched from their index antidepressant and later cycled on and off treatment during the study period $(\$ 12,244$ PPPY in pattern 3c followed by $\$ 11,039$ PPPY in pattern 3b in the switch cohort; Fig. 4b). MDD-related pharmacy costs and mental health-related medical costs contributed minimally to the overall cost burden. 
Table 2 Sociodemographic and clinical characteristics of patients at baseline

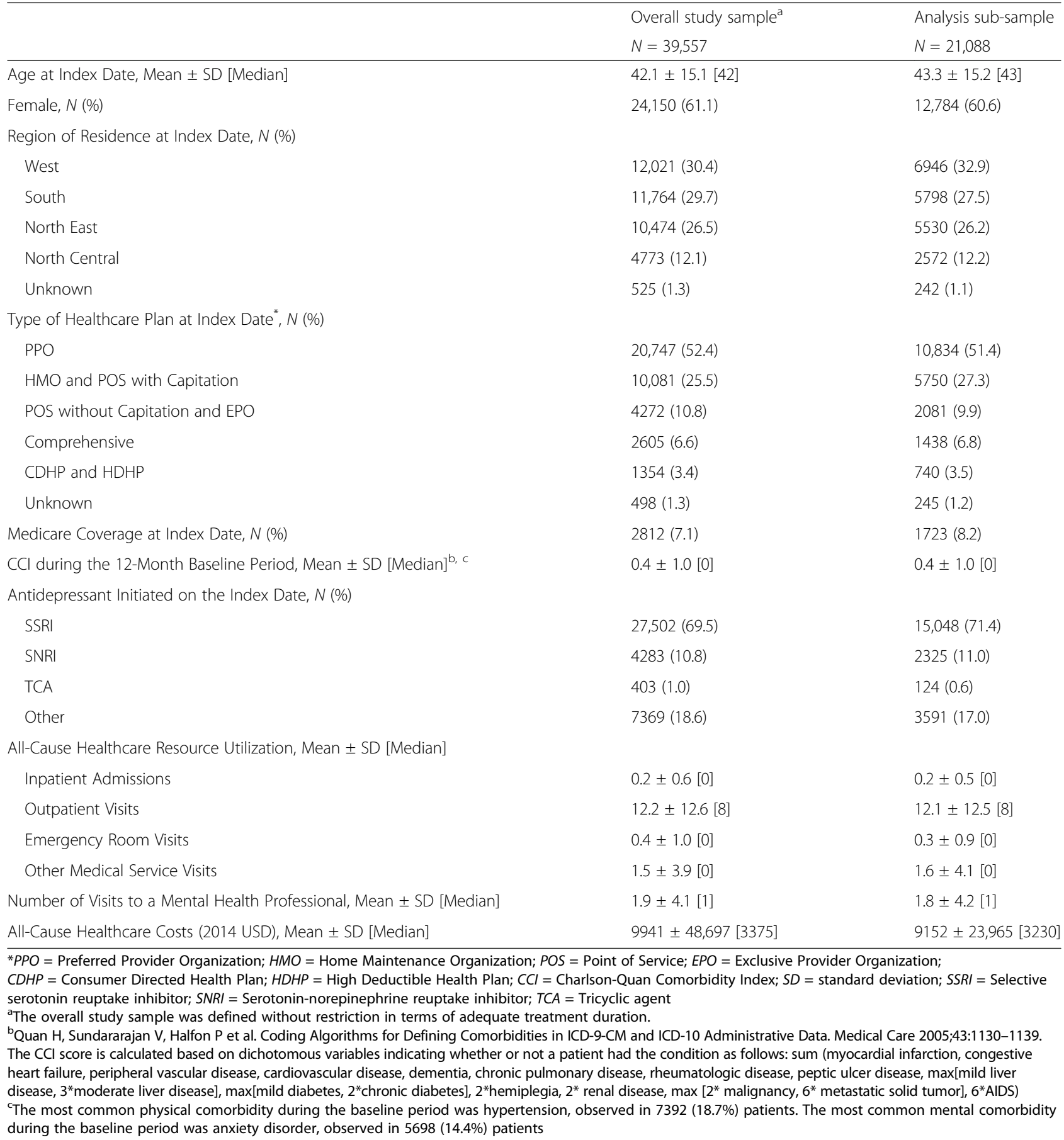

\section{Discussion}

This retrospective study provides the first comprehensive characterization of real-world, long-term treatment patterns following initiation of first-line antidepressant therapy among patients with MDD using data from a large US administrative database. This work further represents a novel contribution to the scientific literature by reporting on the economic burden associated with recommended treatment patterns commonly observed in clinical practice in the US.

Our study included a total of 39,557 patients with MDD who were observed for an average of 4.1 years after initiation of first-line antidepressant therapy. Half of the patients had adequate treatment duration in each line of therapy before the occurrence of a treatment change and were included in the analysis sub-sample. 
Table 3 Treatment cohorts and most common treatment patterns within each cohort

\begin{tabular}{|c|c|}
\hline & $N(\%)^{a}$ \\
\hline Total Number of Patients - Analysis Sub-Sample & $21,088(100 \%)$ \\
\hline Number of patients with 3 most common treatment patterns within each cohort & $14,882(70.6 \%)$ \\
\hline Discontinuation (Cohort 1) & $10,358(49.1)$ \\
\hline Pattern 1a: Discontinuation $\rightarrow$ Remained Untreated & $4833(22.9)$ \\
\hline Pattern 1b: Discontinuation $\rightarrow$ Resumption $\rightarrow$ Discontinuation $\rightarrow$ Resumption & $2364(11.2)$ \\
\hline Pattern 1c: Discontinuation $\rightarrow$ Resumption $\rightarrow$ Discontinuation $\rightarrow$ Remained Untreated & $1158(5.5)$ \\
\hline other patterns & $2003(9.5)$ \\
\hline Dose Escalation (Cohort 2) & $7879(37.4)$ \\
\hline Pattern 2a: Escalation $\rightarrow$ Discontinuation $\rightarrow$ Remained Untreated & $1918(9.1)$ \\
\hline Pattern 2b: Escalation $\rightarrow$ Discontinuation $\rightarrow$ Resumption $\rightarrow$ Discontinuation & $1866(8.8)$ \\
\hline Pattern 2c: Escalation $\rightarrow$ Persistence & $1104(5.2)$ \\
\hline other patterns & $2991(14.2)$ \\
\hline Switch (Cohort 3) & $1390(6.6)$ \\
\hline Pattern 3a: Switch $\rightarrow$ Discontinuation $\rightarrow$ Remained Untreated & $275(1.3)$ \\
\hline Pattern 3b: Switch $\rightarrow$ Discontinuation $\rightarrow$ Resumption $\rightarrow$ Discontinuation & $198(0.9)$ \\
\hline Pattern 3c: Switch $\rightarrow$ Escalation $\rightarrow$ Discontinuation $\rightarrow$ Resumption & $185(0.9)$ \\
\hline other patterns & $732(3.5)$ \\
\hline Persistence (Cohort 4) & $732(3.5)^{\mathrm{b}, \mathrm{c}}$ \\
\hline Combination (Cohort 5) & $588(2.8)^{\mathrm{c}}$ \\
\hline Pattern 5a: Combination $\rightarrow$ Switch/Drop $\rightarrow$ Discontinuation $\rightarrow$ Resumption & $82(0.4)$ \\
\hline Pattern 5b: Combination $\rightarrow$ Escalation $\rightarrow$ Switch/Drop $\rightarrow$ Combination & $58(0.3)$ \\
\hline Pattern 5c: Combination $\rightarrow$ Switch/Drop $\rightarrow$ Combination $\rightarrow$ Switch/Drop & $60(0.3)$ \\
\hline other patterns & $388(1.8)$ \\
\hline Augmentation (Cohort 6) & $141(0.7)^{c}$ \\
\hline Pattern 6a: Augmentation $\rightarrow$ Switch/Drop $\rightarrow$ Augmentation $\rightarrow$ Switch/Drop & $19(0.1)$ \\
\hline Pattern 6b: Augmentation $\rightarrow$ Switch/Drop $\rightarrow$ Discontinuation $\rightarrow$ Resumption & $18(0.1)$ \\
\hline Pattern 6c: Augmentation $\rightarrow$ Persistence & $12(0.1)$ \\
\hline other patterns & $83(0.4)$ \\
\hline
\end{tabular}

${ }^{a}$ All percentages were computed among the total number of patients in the analysis sub-sample $(N=21,088)$

${ }^{\mathrm{b}}$ The mean and median duration of treatment for the persistence cohort was 37.9 and 33.6 months, respectively

${ }^{\mathrm{C}}$ Treatment cohorts representing $<5 \%$ of the analysis sub-sample were not further analyzed due to limited sample size (decided a priori)

Following initiation of first-line therapy, only 3.5\% of patients persisted on their initial antidepressant throughout the study period. The most prevalent treatment change following initiation of first-line therapy was treatment discontinuation, which occurred in approximately half $(49.1 \%)$ of the analysis sub-sample (median time to discontinuation was 23 weeks). Further analysis within this cohort revealed that most patients discontinued their index antidepressant and remained untreated or cycled on and off treatment throughout the study period. Among patients who continued treatment, dose escalation (37.4\% of patients) and treatment switch $(6.6 \%$ of patients) were the most common treatment changes following initiation of first-line therapy. Additionally, our study showed that treatment patterns representing a cycling on and off treatment in the switch cohort were associated with the highest mean total healthcare costs. It should be noted that the rate of switch reported in the current study reflects the first treatment change only; accordingly, the overall rate of switch and associated costs throughout the life cycle of treatment is presumably higher.

The study findings show that, in real-world practice, despite the fact that US guidelines recommend treatments to be administered for four to eight weeks before considering a patient as responsive or unresponsive to treatment, a sizable number of patients experience multiple treatment changes after a treatment duration that may not be sufficient to adequately evaluate treatment response $[11,23]$. Hence, this study further underscores the need for improved long-term, personalized psychiatric management of patients aimed at yielding the best treatment outcomes possible. 

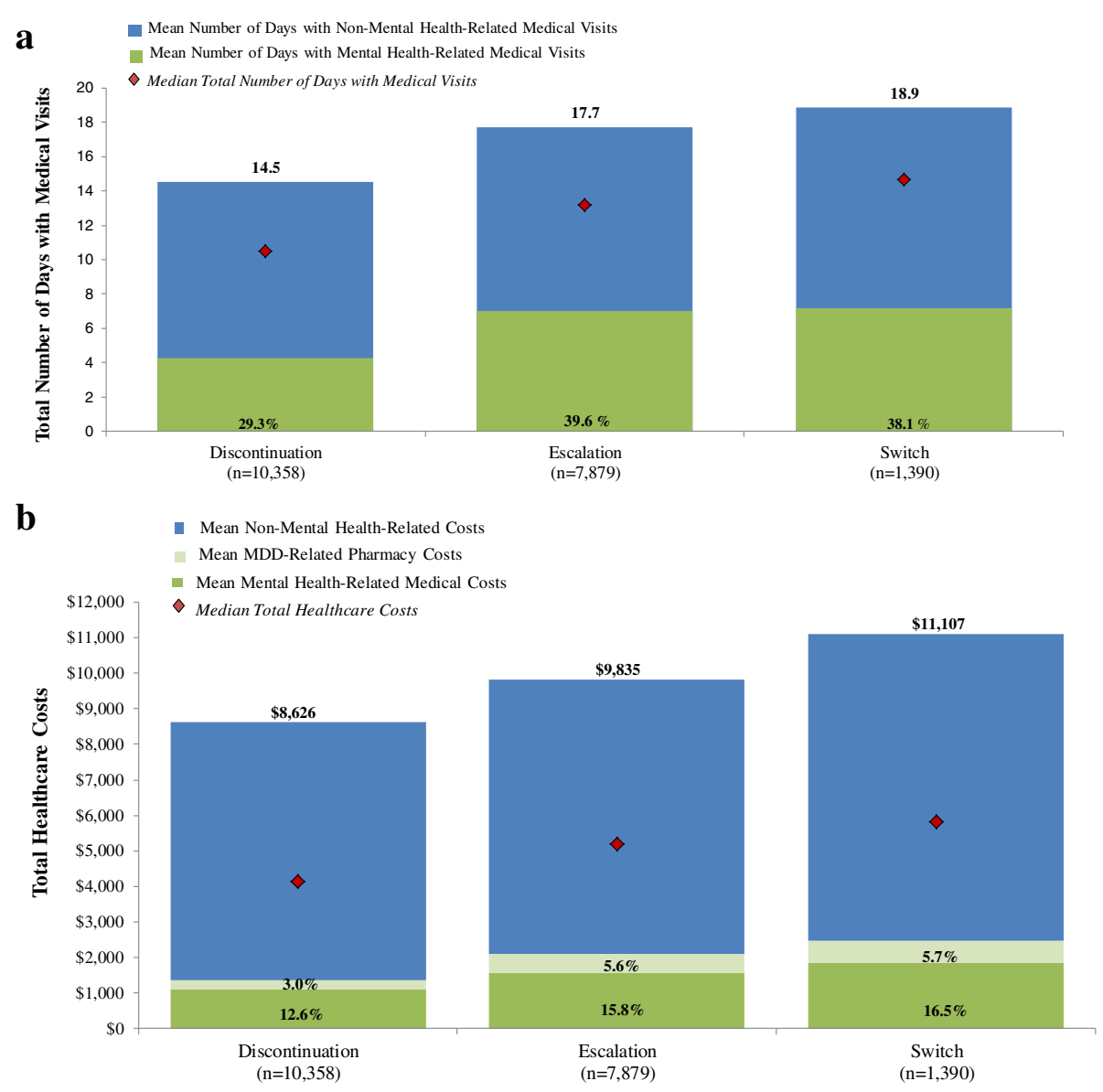

Fig. 3 a Healthcare resource utilization PPPY during the study period - treatment cohort-level [1]. Data on HCRU are presented for the cohorts that each comprised more than $5 \%$ of the analysis sub-sample (decided a priori) [2]. A total number of 14.5 days with medical visits per year in the discontinuation cohort indicates that there was an average of one visit per month, approximately (this interpretation applies across cohorts) [3]. Mean follow-up was 49.6 months in the discontinuation cohort, 49.1 months in the dose escalation cohort, and 50.6 months in the switch cohort [4]. Percentage within each bar represents proportion of days with mental health-related medical visits. b Total healthcare costs PPPY during the study period - treatment cohort-level [1]. Data on costs are presented for the cohorts that each comprised more than 5\% of the analysis sub-sample (decided a priori) [2]. Mean follow-up was 49.6 months in the discontinuation cohort, 49.1 months in the dose escalation cohort, and 50.6 months in the switch cohort [3]. Percentages within each bar represent proportions of mental health-related medical costs and MDD-related pharmacy costs

Although most practice guidelines state that pharmacological therapy of patients with MDD typically includes three phases - acute phase (6-8 weeks), continuation phase (16-20 weeks), and maintenance phase for chronic and/or recurrent MDD [23] - our results show that, in real-world practice, a considerable proportion of patients do not reach the end of the continuation phase due to treatment discontinuation. Consequently, a significant number of patients could benefit from continuing therapy over a longer period of time assuming no major safety and tolerability issues are of concern.

The high rate of discontinuation in our study is consistent with the high discontinuation rate reported in a recent study by Jung et al. [24]. In this study, over $70 \%$ of patients discontinued antidepressants after 6 months (including patients who switched). While some patients may discontinue therapy due to having achieved remission, treatment side effects, inadequate patient follow-up, patient concern about medications and fear of addiction, physician-patient miscommunication, misperception of the benefits of treatment, or patient perception that treatment is no longer needed are also common reasons for discontinuation [25-31]. Indeed, as response to first-line therapy is often less than optimal [16-19], it is likely that the discontinuation cohort in our study includes non-responders and non-remitters. Furthermore, some of the symptoms that characterize MDD - such as difficulty planning, disorganization, distraction, and other cognitive impairments - are also likely to contribute to poor persistence on first-line 

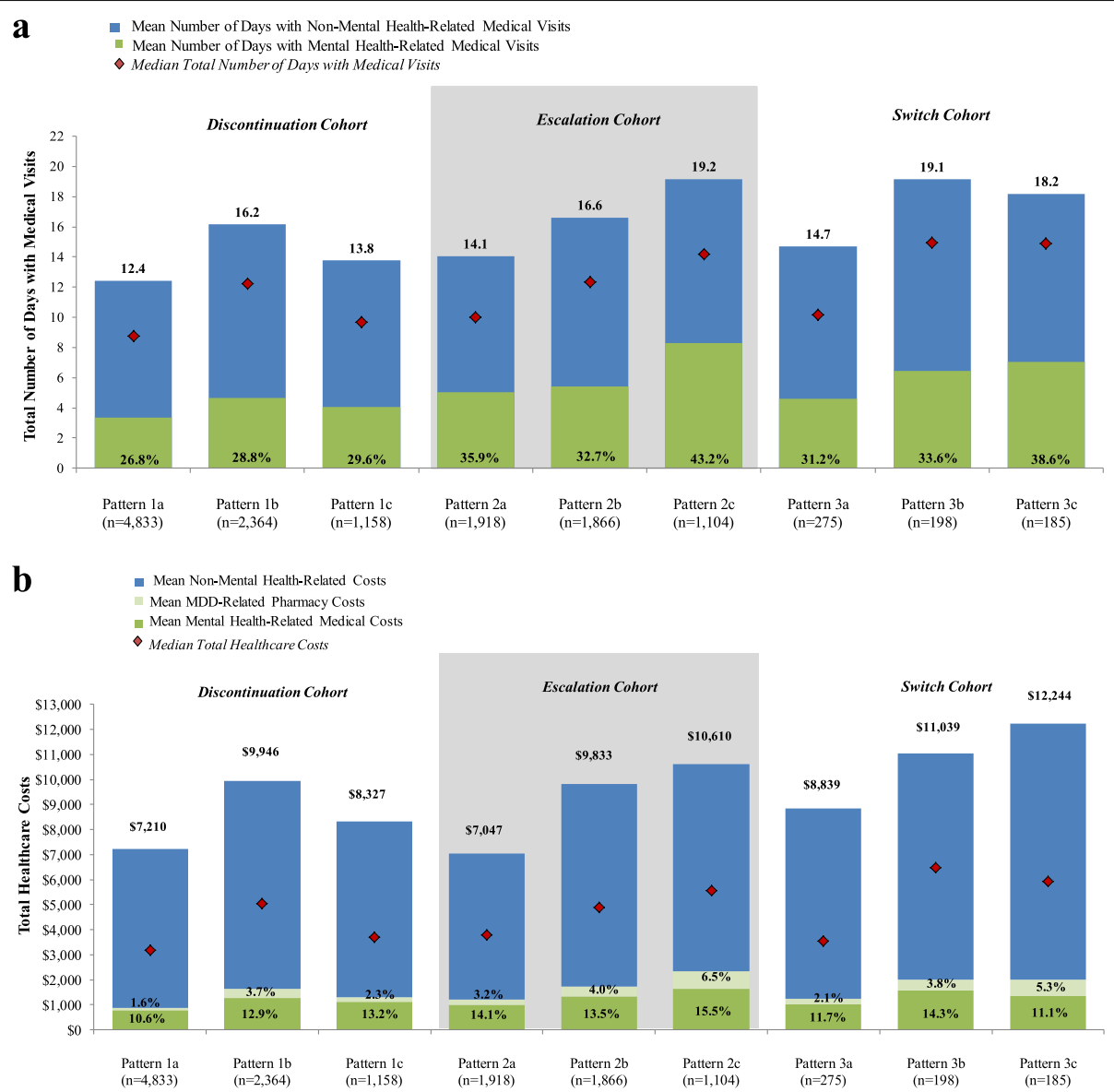

Fig. 4 a Healthcare resource utilization PPPY during the study period - treatment pattern-level. Pattern 1a: Discontinuation $\rightarrow$ Remained Untreated Pattern 1b: Discontinuation $\rightarrow$ Resumption $\rightarrow$ Discontinuation $\rightarrow$ Resumption Pattern 1c: Discontinuation $\rightarrow$ Resumption $\rightarrow$ Discontinuation $\rightarrow$ Remained Untreated Pattern 2a: Escalation $\rightarrow$ Discontinuation $\rightarrow$ Remained Untreated Pattern 2b: Escalation $\rightarrow$ Discontinuation $\rightarrow$ Resumption $\rightarrow$ Discontinuation Pattern 2c: Escalation $\rightarrow$ Persistence Pattern 3a: Switch $\rightarrow$ Discontinuation $\rightarrow$ Remained Untreated Pattern 3b: Switch $\rightarrow$ Discontinuation $\rightarrow$ Resumption $\rightarrow-$ Discontinuation Pattern 3c: Switch $\rightarrow$ Escalation $\rightarrow$ Discontinuation $\rightarrow$ Resumption.[1] Data on HCRU are presented only for the three most prevalent patterns within each cohort that comprised more than $5 \%$ of the analysis sub-sample (decided a priori). [2] Percentage within each bar represents proportion of days with mental health-related medical visits. b Total healthcare costs PPPY during the study period - treatment pattern-level. Pattern 1a: Discontinuation $\rightarrow$ Remained Untreated Pattern 1b: Discontinuation $\rightarrow$ Resumption $\rightarrow$ Discontinuation $\rightarrow$ Resumption Pattern 1c: Discontinuation $\rightarrow$ Resumption $\rightarrow$ Discontinuation $\rightarrow$ Remained Untreated Pattern 2a: Escalation $\rightarrow$ Discontinuation $\rightarrow$ Remained Untreated Pattern 2b: Escalation $\rightarrow$ Discontinuation $\rightarrow$ Resumption $\rightarrow$ Discontinuation Pattern 2c: Escalation $\rightarrow$ Persistence Pattern 3a: Switch $\rightarrow$ Discontinuation $\rightarrow$ Remained Untreated Pattern 3b: Switch $\rightarrow$ Discontinuation $\rightarrow$ Resumption $\rightarrow$ Discontinuation Pattern 3c: Switch $\rightarrow$ Escalation $\rightarrow$ Discontinuation $\rightarrow$ Resumption [1] Data on costs are presented only for the three most prevalent patterns within each cohort that comprised more than $5 \%$ of the analysis sub-sample (decided a priori) [2]. Percentages within each bar represent proportions of mental health-related medical costs and MDD-related pharmacy costs

antidepressant and frequent cycling on and off treatment. Although the reasons for therapy discontinuation were not available, and that it is possible that a number of patients discontinued due to successful treatment, the high discontinuation rates and the relatively short period of time within which patients discontinued their firstline antidepressant also suggest that there may be a need to improve long-term treatment persistence in some patients with MDD.

Dose escalation and switching to another antidepressant or to an atypical antipsychotic were found to be the most common treatment changes among patients who continued treatment after first-line therapy. This finding suggests a physician's preference for antidepressant drug monotherapy versus adding or combining therapies. Although the choice of the next treatment step is likely to depend on the initial treatment response (i.e., partial vs. no response) [11, 32], a physician's preference for monotherapy in the US could reflect an effort to avoid potential drug interactions and to maximize compliance by minimizing the number of MDD pharmacotherapies prescribed.

In terms of HCRU and costs, our results indicate that treatment patterns representing a cycling on and off treatment in the switch cohort were associated with the greatest total healthcare costs PPPY over the long-term 
follow-up. This may suggest that patients who commonly switch and continually cycle through different treatments might be particularly difficult-to-treat patients, thus driving medical costs up. It should, however, be noted that the treatment patterns observed in this study, along with their outcomes, may not reflect optimal treatment paths and can have grave consequences on patient responses and treatment costs. For example, it may be more appropriate to switch a patient from their first line of treatment rather than escalating their initial dose; this may, for some patients, result in lower burden and reduced long-term costs if treatment selection is appropriately determined early in the treatment path, i.e., it may be more costly and burdensome if a patient remains on a treatment that fails to be effective rather than switching to a different, more optimal, treatment [33]). Results for HCRU and costs also suggest that a relatively high proportion of the medical services and costs are not related to mental health. These results are consistent with prior studies. For example, Simon et al. (1995) reported that $66 \%$ to $71 \%$ of the total direct healthcare costs were not related to antidepressant or mental health-related visit costs [34]. These findings may reflect HCRU and costs associated with the management of other concurrent comorbidities, complications, or unrelated conditions worth evaluating in future studies. Despite the high direct healthcare costs PPPY reported in this study, these costs likely reflect an underestimate of the overall economic burden reported for MDD in the US. Indeed, a previously published US study estimated that only half of the economic burden of MDD was attributable to direct healthcare costs, with the other half attributable to worker absenteeism and presenteeism [10].

Mention of the methodological limitations of this study is warranted. First, treatment cohorts and patterns were identified based on claims for a filled prescription, which does not guarantee that the medication was consumed by the patient or account for changes in prescribed therapy. Second, a patient's medical history may influence treatment decisions and subsequent treatment patterns and outcomes. However, disease severity, clinical characteristics and reasons underlying specific treatment patterns are not available in claims databases. Further analyses using a different data source or data linkage with patients' medical records is warranted to better understand treatment decisions resulting in the patterns observed. Third, the study sample was limited to privately insured employees and their dependents. All patients included in our study sample were required to meet pre-determined inclusion and exclusion criteria (e.g., to have at least two billing claims for MDD within a pre-specified time period and to initiate the index antidepressant in monotherapy as first-line treatment).
Accordingly, the results from our study may not be generalizable to the overall MDD patient population in the US. Fourth, our study was descriptive in nature and no statistical comparisons between treatment cohorts/patterns were conducted. However, it is possible that patients across treatment cohorts/patterns have differential disease profiles. Patients may have also undergone concomitant non-pharmacological therapy, such as psychotherapy, which may influence treatment decisions and outcomes. Non-pharmacological treatment like cognitive behavioral therapy was not evaluated as part of the current study. Further analyses are warranted to better understand reasons for specific treatment changes/patterns and to compare outcomes between treatment cohorts/patterns, including cohorts receiving concomitant psychotherapy. Fifth, the current study identifies dose escalation but did not assess whether the dosage prescribed was within the therapeutic range (a determination that would require that the dose prescribed is indeed suitable for a particular patient). Additional analyses would be needed to analyze the impact of adequate/inadequate dosing on outcomes and costs. Sixth, this study was designed to evaluate MDD patients with treatment patterns involving antidepressant and antipsychotic therapies. It is, however, possible that some patients with MDD also receive other treatments such as lithium and thyroid hormones as part of their augmentation therapy. Other therapies such as lithium and thyroid hormones, for example, were not analyzed in our study as patients augmenting with these agents may require them for the treatment of non-MDD conditions (e.g., lithium for bipolar disorder and thyroid hormones for conditions related to hormonal imbalance) - claims data do not include information to confirm the reason for which a treatment is prescribed. Finally, administrative claims databases are subject to coding errors and data omissions.

\section{Conclusions}

This US study provided the first comprehensive characterization of real-world treatment patterns and associated HCRU and costs following initiation of first-line therapy with an antidepressant in patients with MDD. The results showed that an exceptionally small number of patients persisted on their first-line antidepressant throughout the study period. In contrast, nearly half of the analysis sub-sample discontinued therapy following first-line antidepressant, making discontinuation the most common initial treatment change. Discontinuation and resumption of treatment within the switch cohort was generally associated with the highest healthcare costs. In conclusion, this study underscores several challenges in effectively treating MDD and an urgent need for personalized patient management aimed at improving quality of care and reducing overall burden. 


\section{Abbreviations}

AD: antidepressant; CCl: Charlson-Quan comorbidity index; CDHP: consumer directed health plan; DX: diagnosis; EPO: exclusive provider organization; HCRU: healthcare resource utilization; HDHP: high deductible health plan; HMO: Home Maintenance Organization; MarketScan: Truven Health Analytics MarketScan ${ }^{\oplus}$ Databases; MDD: major depressive disorder; POS: point of service; PPO: preferred provider organization; PPPY: per-patient-per-year; SD: standardized deviation; SNRI: serotonin-norepinephrine reuptake inhibitor; SSRI: selective serotonin reuptake inhibitor; TCA: tricyclic agent; US: United States; USD: United States dollar

\section{Acknowledgements}

The authors would like to thank Cinzia Metallo for medical writing assistance.

\section{Funding}

This study was sponsored by the Takeda Pharmaceutical Company, Ltd. and Lundbeck A/S. The title of the funded research study is: Treatment Patterns, Healthcare Resource Utilization, and Costs Following First-Line Antidepressant Treatment in Major Depressive Disorder.

\section{Availability of data and materials}

The data that support the findings of this study are covered under a license agreement and thus cannot be made public and/or shared. Access to these data are available to any interested party(ies) for a fee set by Truven Health Analytics (https://marketscan.truvenhealth.com/ marketscanportal/).

\section{Authors' contributions}

GG and VP conceptualized the initial study design and developed the initia draft of the manuscript. AG, MZ, WJ, GN, EM, and CF provided constructive feedback on the initial study design. $G G, A G$, and $M Z$ analyzed the data. $G G$, $A G, M Z, W J, G N, E M, C F$, and VP interpreted the data, provided constructive feedback during manuscript development, and read and approved the final manuscript. The design, analysis, reporting of findings, and intent to publish represent the views and opinions of the authors and do not represent the views and opinions of their respective organizations/companies. All authors read and approved the final manuscript.

\section{Competing interests}

This study was funded by the Takeda Pharmaceutical Company, Ltd. and Lundbeck A/S. Perez V. and Jacobson W. are employees of Takeda Development Center Americas, Inc. At the time this research was conducted and completed, Merikle E. and Nomikos G. were employed by Takeda Development Center Americas, Inc. Gauthier G., Guerin A., and Zhdanava M. are employees of Analysis Group, Inc., which received consultancy fees from Takeda. Francois $C$ is an employee of Lundbeck A/S. Medical writing assistance was provided by Cinzia Metallo, an employee of Analysis Group, Inc.

\section{Consent for publication}

Not applicable.

\section{Ethics approval and consent to participate}

Data are de-identified and comply with the Health Insurance Portability and Accountability Act [22]. No institutional review board approval was necessary for the current study.

\section{Publisher's Note}

Springer Nature remains neutral with regard to jurisdictional claims in published maps and institutional affiliations.

\section{Author details}

${ }^{1}$ Analysis Group, Inc., Montreal, QC, Canada. ${ }^{2}$ Takeda Development Center Americas, Inc., One Takeda Parkway, Deerfield, IL 60015, USA. ${ }^{3}$ Sage Therapeutics, Cambridge, MA, USA. ${ }^{4}$ Covance Inc., Gaithersburg, MD, USA. ${ }^{5}$ Lundbeck S.A.S, Deerfield, IL, USA.
Received: 21 November 2016 Accepted: 9 June 2017

Published online: 19 June 2017

\section{References}

1. American Psychiatric Association. Diagnostic and Statistical Manual of Mental Disorders, Fifth ed. Arlington, VA: American Psychiatric Association Press 2013.

2. Kennedy SH. Core symptoms of major depressive disorder: relevance to diagnosis and treatment. Dialogues in Clin Neurosci. 2008;10(3):271-7.

3. (NIMH) NloMH. Major Depression Among Adults. http://www.nimh.nih.gov/ health/statistics/prevalence/major-depression-among-adults.shtml. Accessed August 2016.

4. World Health Organization. Depression Fact Sheet. 2016. http://www.who. int/mediacentre/factsheets/fs369/en/. Accessed July 2016.

5. Substance Abuse and Mental Health Services. Behavioral Health Trends in the United States: Results from the 2014 National Survey on Drug Use and Healths. http://www.samhsa.gov/data/sites/default/files/NSDUH-FRR1-2014/ NSDUH-FRR1-2014.htm\#idtextanchor079. Accessed July 2016.

6. Saltiel PF, Silvershein DI. Major depressive disorder: mechanism-based prescribing for personalized medicine. Neuropsychiatric Dis Treat. 2015;11(875-888.

7. Gartlehner G, Gaynes BN, Amick HR, Asher G, Morgan LC, Coker-Schwimmer E, et al. Nonpharmacological versus pharmacological treatments for adult patients with major depressive disorder. In: AHRQ Comparative effectiveness reviews. ed. Rockville (MD): Agency for Healthcare Research and Quality (US); 2015.

8. Kessler RC. The costs of depression. Psychiatr Clin North Am. 2012;35(1):1-14.

9. Lépine J-P, Briley M. The increasing burden of depression. Neuropsychiatric Dis Treat. 2011;7(Suppl 1):3-7.

10. Greenberg PE, Fournier AA, Sisitsky T, Pike $C T$, Kessler RC. The economic burden of adults with major depressive disorder in the United States (2005 and 2010). J Clin Psychiatry. 2015;76(2):155-62.

11. American Psychiatric Association. Work Group on Major Depressive Disorder Practice guideline for the treatment of patients with major depressive disorder 3rd ed. 2010. https://psychiatryonline.org/pb/assets/raw/sitewide/ practice_guidelines/guidelines/mdd.pdf. Accessed June 2016.

12. Armstrong C. APA releases guideline on treatment of patients with major depressive disorder. Am Fam Physician. 2011;83(10):1219-27.

13. Qaseem A, Snow V, Denberg TD, Forciea MA, Owens DK. Using secondgeneration antidepressants to treat depressive disorders: a clinical practice guideline from the American College of Physicians. Ann Intern Med. 2008;149(10):725-33.

14. Madhukar HT, Ella JD. Treatment strategies to improve and sustain remission in major depressive disorder. Dialogues Clin Neurosci. 2008;10(4):377-84

15. Blier P, El Mansari M. Serotonin and beyond: therapeutics for major depression. Phil Trans R Soc B: Biological Sciences. 2013;368(1615):20120536.

16. Connolly KR, Thase ME. If at first you don't succeed: a review of the evidence for antidepressant augmentation, combination and switching strategies. Drugs. 2011;71(1):43-64.

17. Sinyor M, Schaffer A, Levitt A. The sequenced treatment alternatives to relieve depression (STAR*D) trial: a review. Can J Psychiatr. 2010;55(3):126-35.

18. Papakostas GI. Managing partial response or nonresponse. Switching, augmentation, and combination strategies for major depressive disorder. J Clin Psychiatry. 2009;70(Suppl 6):16-25.

19. Gaynes BN, Warden D, Trivedi MH, Wisniewski SR, Fava M, Rush AJ. What did STAR*D teach us? Results from a large-scale, practical, clinical trial for patients with depression. Psychiatr Serv. 2009;60(11):1439-45.

20. Garcia-Toro M, Medina E, Galan JL, Gonzalez MA, Maurino J. Treatment patterns in major depressive disorder after an inadequate response to first-line antidepressant treatment. BMC Psychiatry. 2012;12(1):1-6.

21. Truven Health Analytics. Truven Health MarketScan Research Databases. http://truvenhealth.com/portals/0/assets/HP_11517_0912_ MarketScanResearchDatabasesForHP_SS_WEB.pdf. Accessed on July 2016.

22. Truven Health Analytics. Health Research Data for the Real World: the MarketScan Databases. http://truvenhealth.com/portals/0/assets/PH_11238_ 0612_TEMP_MarketScan_WP_FINAL.pdf. Accesed on June 2017.

23. American Psychiatric Association. Practice Guideline for the Treatment of Patients with Major Depressive Disorder. 2nd ed. 2000. http://networkhealth.org/uploadedFiles/pdfs/clinical_guidelines/guidelines_depression_ treatment.pdf. Accessed June 2016. 
24. Jung W-Y, Jang S-H, Kim S-G, Jae Y-M, Kong B-G, Kim H-C, et al. Times to discontinue antidepressants over 6 months in patients with major depressive disorder. Psychiatry Investig. 2016;13(4):440-6.

25. Sansone RA, Sansone LA. Antidepressant adherence: are patients taking their medications? Innov Clin Neurosci. 2012;9(5-6):41-6.

26. Fortney JC, Pyne JM, Edlund MJ, Stecker T, Mittal D, Robinson DE, et al. Reasons for antidepressant nonadherence among veterans treated in primary care clinics. J Clin Psychiatry. 2011;72(6):827-34.

27. Bulloch AG, Patten SB. Non-adherence with psychotropic medications in the general population. Soc Psychiatry Psychiatr Epidemiol. 2010;45(1):47-56.

28. Kennedy J, Tuleu I, Mackay K. Unfilled prescriptions of medicare beneficiaries: prevalence, reasons, and types of medicines prescribed. J Manag Care Pharm. 2008;14(6):553-60.

29. Chakraborty K, Avasthi A, Kumar S, Grover S. Attitudes and beliefs of patients of first episode depression towards antidepressants and their adherence to treatment. Soc Psychiatry Psychiatr Epidemiol. 2009;44(6):482-8.

30. Hoencamp E, Stevens A, Haffmans J. Patients' attitudes toward antidepressants. Psychiatr Serv. 2002;53(9):1180-1.

31. Masand PS. Tolerability and adherence issues in antidepressant therapy. Clin Ther. 2003;25(8):2289-304.

32. Fredman SJ, Fava M, Kienke AS, White CN, Nierenberg AA, Rosenbaum JF. Partial response, nonresponse, and relapse with selective serotonin reuptake inhibitors in major depression: a survey of current "next-step" practices. J Clin Psychiatry. 2000;61(6):403-8.

33. Chekroud AM, Zotti RJ, Shehzad Z, Gueorguieva R, Johnson MK, Trivedi MH, et al. Cross-trial prediction of treatment outcome in depression: a machine learning approach. The Lancet Psychiatry. 3(3):243-50.

34. Simon GE, VonKorff M, Heiligenstein JH, Revicki DA, Grothaus L, Katon W, et al. Initial antidepressant choice in primary care. Effectiveness and cost of fluoxetine vs tricyclic antidepressants. JAMA. 1996;275(24):1897-902.

\section{Submit your next manuscript to BioMed Central and we will help you at every step:}

- We accept pre-submission inquiries

- Our selector tool helps you to find the most relevant journal

- We provide round the clock customer support

- Convenient online submission

- Thorough peer review

- Inclusion in PubMed and all major indexing services

- Maximum visibility for your research

Submit your manuscript at www.biomedcentral.com/submit

) Biomed Central 